\title{
Salami-slicing and plagiarism: How should we respond?
}

\author{
Martin G. Tolsgaard ${ }^{1} \cdot$ Rachel Ellaway $^{2} \cdot$ Nikki Woods $^{3} \cdot$ Geoff Norman $^{4}$
}

(c) Springer Nature B.V. 2019

One particularly odious task that every journal editor must confront from time to time is the investigation of a case of scientific fraud. We are not talking about blatant cases, where data are falsified; these are almost impossible to detect. Rather, a far more common occurrence is the deliberate use of previously published date and/or text-either the author's own or, more seriously, plagiarized from other authors' publications. Three labels are attached to these acts: Plagiarism—copying from another author's published work; Autoplagiarism-duplication of previously published by the authors without clearly stating that this was the case; Salami-slicing-authors have published parts of a study in multiple papers instead of providing the full story in a single paper. These are not new problems in medical education research (Brice et al. 2009) but the incidence seems to be increasing over the years (Steen 2011). Although these problems have been addressed in recent editorials in medical education journals (Norman 2014; Eva 2017), there seems to be a lack of consensus on how to handle manuscripts that have these problems.

Identifying examples of text copied from elsewhere used to be difficult, as it required extensive cross-referencing. Nowadays, plagiarism detection tools feature in many journals' workflows (including AHSE), so previously published text can be quickly identified along with its provenance. However, while these automated tools can indicate where sentences or fragments arise from other texts, many of these are perfectly legitimate. It is almost impossible to have a paper with no "plagiarized" text based on automated search, and judgment is required to identify the demarcation between legitimate uses of phrases or sentences and clear plagiarism. Similar issues arise with "auto-plagiarism" and salamislicing. When an author uses similar methodology in a series of studies, it is natural that some methodological description may well be common to several papers, Salami slicing for one editor might be seen as programmatic research by others (Eva 2017). Nevertheless, editors need to determine if there is clear evidence of misconduct, whether there are reasons to sanction the authors for this conduct, and if so then how this should be done.

Geoff Norman

norman@mcmaster.ca

1 Copenhagen Academy for Medical Education and Simulation (CAMES), Rigshospitalet and University of Copenhagen, Copenhagen, Denmark

2 Department of Community Health Sciences, University of Calgary, Calgary, AB, Canada

3 Department of Family and Community Medicine, University of Toronto, Toronto, ON, Canada

4 Department of Health Research Methods, Evidence and Impact, McMaster University, Hamilton, ON, Canada 
About 2 years ago, we began to systematically track suspected incidents. To date, 25 manuscripts have been submitted to AHSE where editors have suspected plagiarism (15), auto-plagiarism (5) or salami-slicing (5). Thirteen authors received a warning, 4 manuscripts have been rejected cautioning authors and 8 authors have been blacklisted and not allowed to submit any more manuscripts to the Journal. Of course, it remains to be seen how many of these articles would not be accepted even without issues of plagiarism. However, all of these decisions were made by the editor-in-chief without consultation, and it remains to be seen what kind of agreement would arise among editors. Guidelines do exist, (COPE) (https://publicationethics.org/resources/flowcharts) and articles have appeared in other medical education journals (Brice et al. 2009; Eva 2017; Norman 2014).

To inform journal policy and to provide author guidance for Advances in Health Sciences Education (AHSE), we surveyed our editorial board with respect to how members of the editorial board considered salami-slicing, plagiarism, and auto-plagiarism in terms of scientific conduct and its appropriate consequences.

\section{A study}

We designed an online-survey of possible practices related to these areas and potential actions. Survey items were developed and informed by existing literature on salami-slicing, auto-plagiarism, and plagiarism in health professions education and in accordance with the guidelines for survey development described by Gehlbach et al. (2010). Survey items were generated based on consensus among the study authors and were categorized in three parts; seriousness of action, expected response from editors, and transparency declaration. The survey was sent to all associate editors of Advances in Health Sciences Education. Survey results were presented at the editorial board meeting in August 2018. From this we developed a second questionnaire that included items that involved editor responsibilities and the role of pre-publication of scientific manuscripts. This second questionnaire was sent to AHSE editors in September 2018. Editors received one reminder per questionnaire within 4 weeks of the invitation. Descriptive statistics were performed and extent of plagiarism, auto-plagiarism, salami-slicing as well as their consequences were compared using parametric statistics, when appropriate. Bonferroni corrections were used to adjust for multiple comparisons.

Ethical approval was granted at the University of Calgary (REB17-2407). Survey studies are exempt from ethical approval in Denmark according to national regulations.

Twenty-four of the 25 AHSE associate editors completed both survey rounds (96\%). Substantial plagiarism and auto-plagiarism were perceived as 'a major issues' or 'unacceptable academic behavior' by at least half of the editors when authors failed to provide any referencing to the publication, from which the text was plagiarized (Table 1). On the contrary, if authors provided appropriate referencing, the majority of editors perceived varying degrees of plagiarism and auto-plagiarism to be 'very minor' or 'minor issues' (Table 1).

\section{Acceptable behaviours}

Plagiarism was less tolerated than auto-plagiarism when authors failed to provide adequate referencing $(\mathrm{t}=-4.3$, d.f. $=19 ; p=0.002$ for 'substantial parts of the text' and $\mathrm{t}=-5.0$, d.f. $=20 ; p<0.01$ for 'sentences'). However, there were no differences in editors' acceptance of plagiarism versus auto-plagiarism when authors provided appropriate referencing 


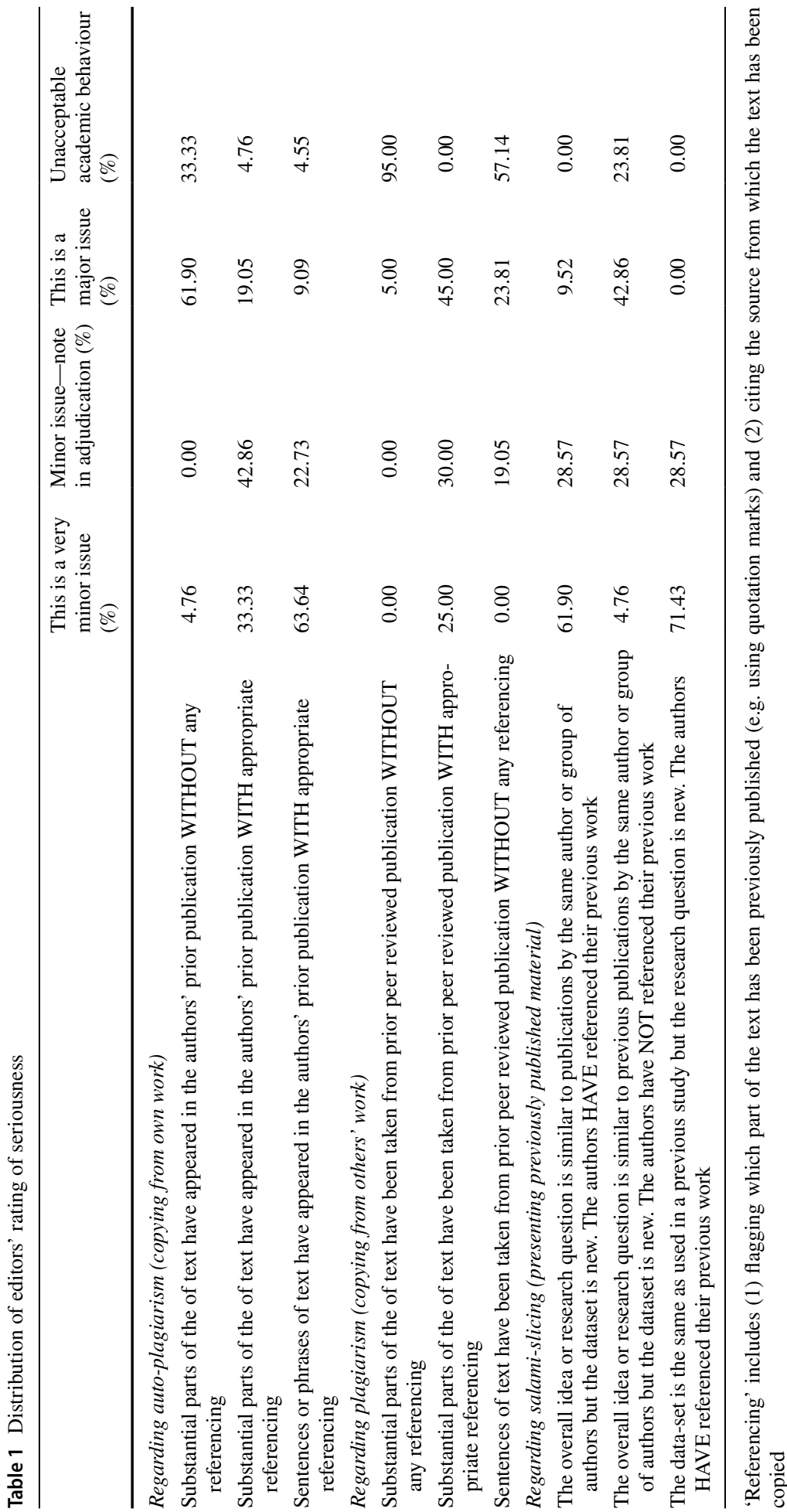


$(\mathrm{t}=-1.2$, d.f. $=19 ; p=1.00$ for 'substantial parts of the text' and $\mathrm{t}=2.7$, d.f. $=20 ; p=1.00$ for 'sentences. Similarly, editors perceived salami-slicing to be a serious matter when authors failed to reference previous publications, but much less so if the prior work was appropriately referenced and difference with present work clearly described.

All editors agreed that publishing abstracts in online conference proceedings was either a minor issue or completely acceptable, and 90\% (22) agreed that reusing material from theses was also acceptable. However, there was more variation in editors' judgment regarding 'pre-publicizing', with the majority of editors being of the opinion that pre-publication is a 'minor problem' or not a problem at all. Free-text responses revealed concerns regarding pre-publication for several reasons, including ethical considerations (breaking the rules of publication by submitting several places), legal considerations (concerns regarding who owns the manuscript), business considerations (pre-publicizing threatens the business model of the journal), and quality concerns (in particular the absence of peer review for pre-published material, which makes it difficult for consumers to tell the difference between content published in real journals versus pre-published papers).

\section{Consequences}

A small proportion of editors (0-15\%) recommended that there should be serious consequences (noted in Table 2) in cases of auto-plagiarism or salami-slicing regardless of whether or not authors provided adequate referencing. Just under half of the respondents recommended serious consequences for plagiarism if authors failed to provide adequate referencing, whereas only one respondent recommended retraction (if already published) in the case of adequate referencing.

\section{Declarations}

$86 \%$ of the respondents agreed that authors should declare potential problems regarding salami-slicing, plagiarism, and auto-plagiarism in the cover letter to editors and $91 \%$ thought it should be mentioned in the manuscript text. None of the editors indicated that omitting such declarations was acceptable.

\section{Editorial responsibility}

$43 \%$ of editors believed that either general or specific rules are needed to guide editors in questions regarding plagiarism and salami-slicing with latitude for individual assessment. $91 \%$ felt they had a shared responsibility with the editorial team to assess whether or not authors have plagiarized or salami-sliced their paper, the other two did not see this as their job and felt responsible only for the academic content.

\section{Discussion}

AHSE associate editors considered auto-plagiarism, plagiarism, and salami-slicing to be a problem in academic publishing. Of the three, plagiarism was considered a more serious issue. The severity of the problem depended on the magnitude of overlap and the extent to which they had or had not declared that they were doing so. There was 


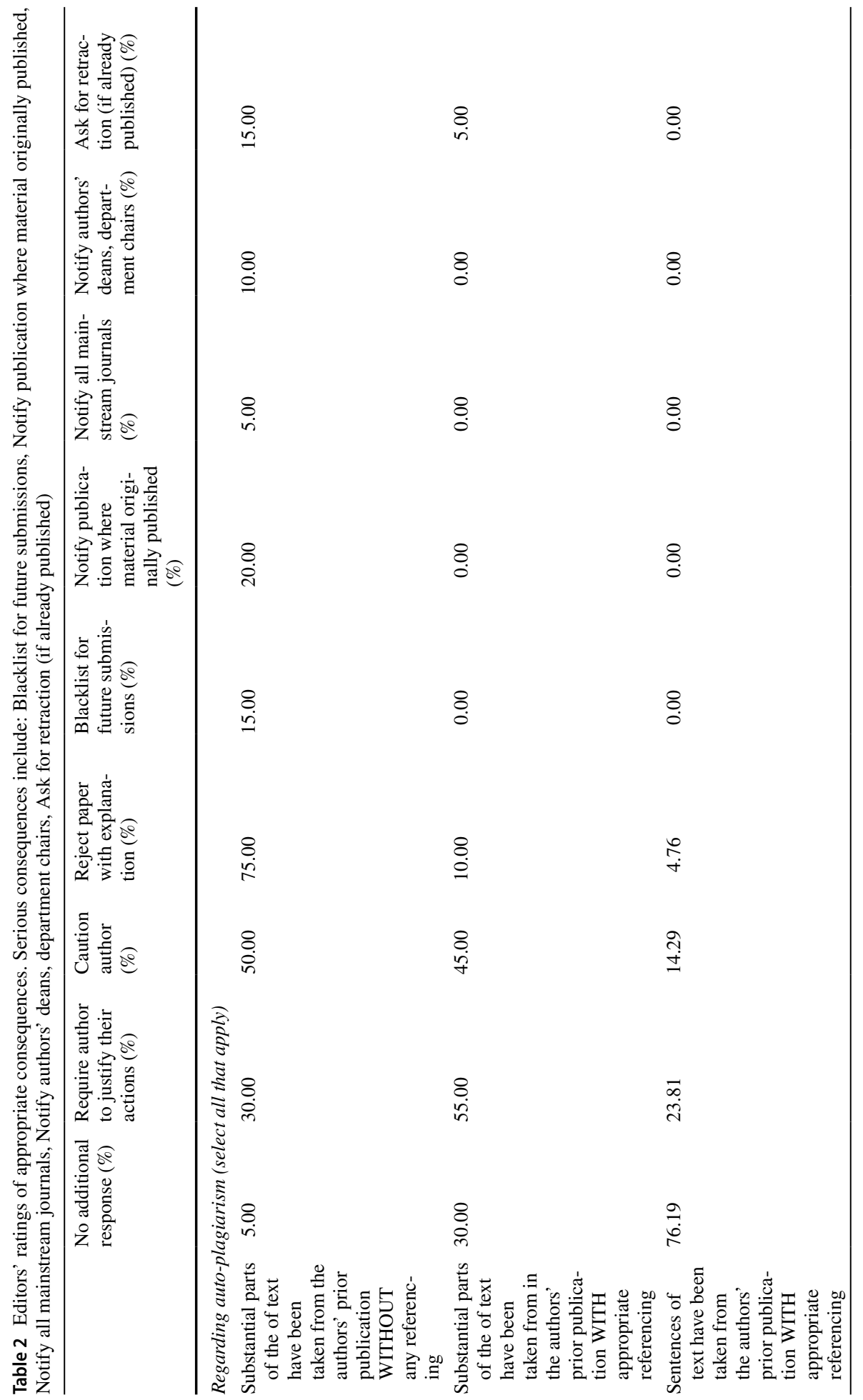




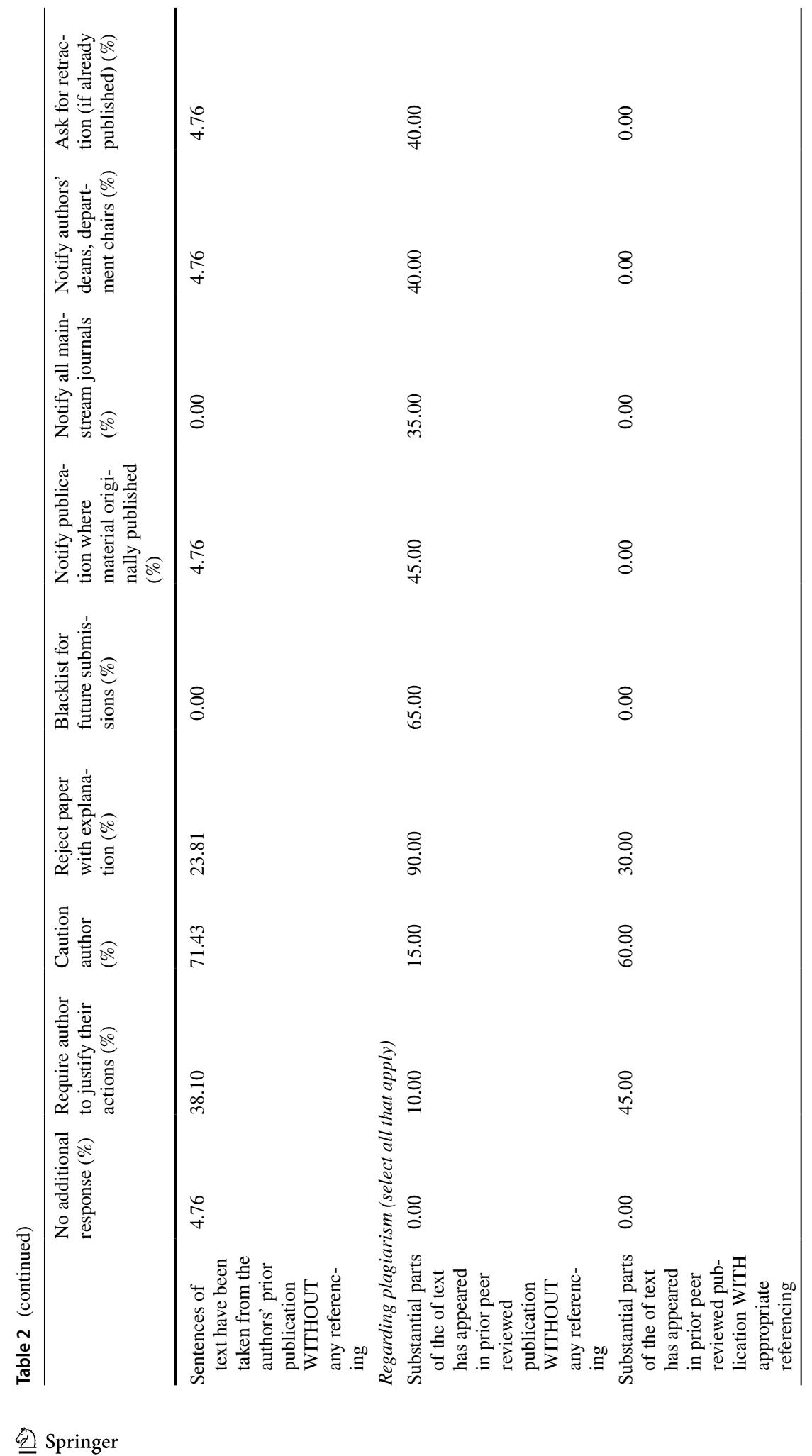




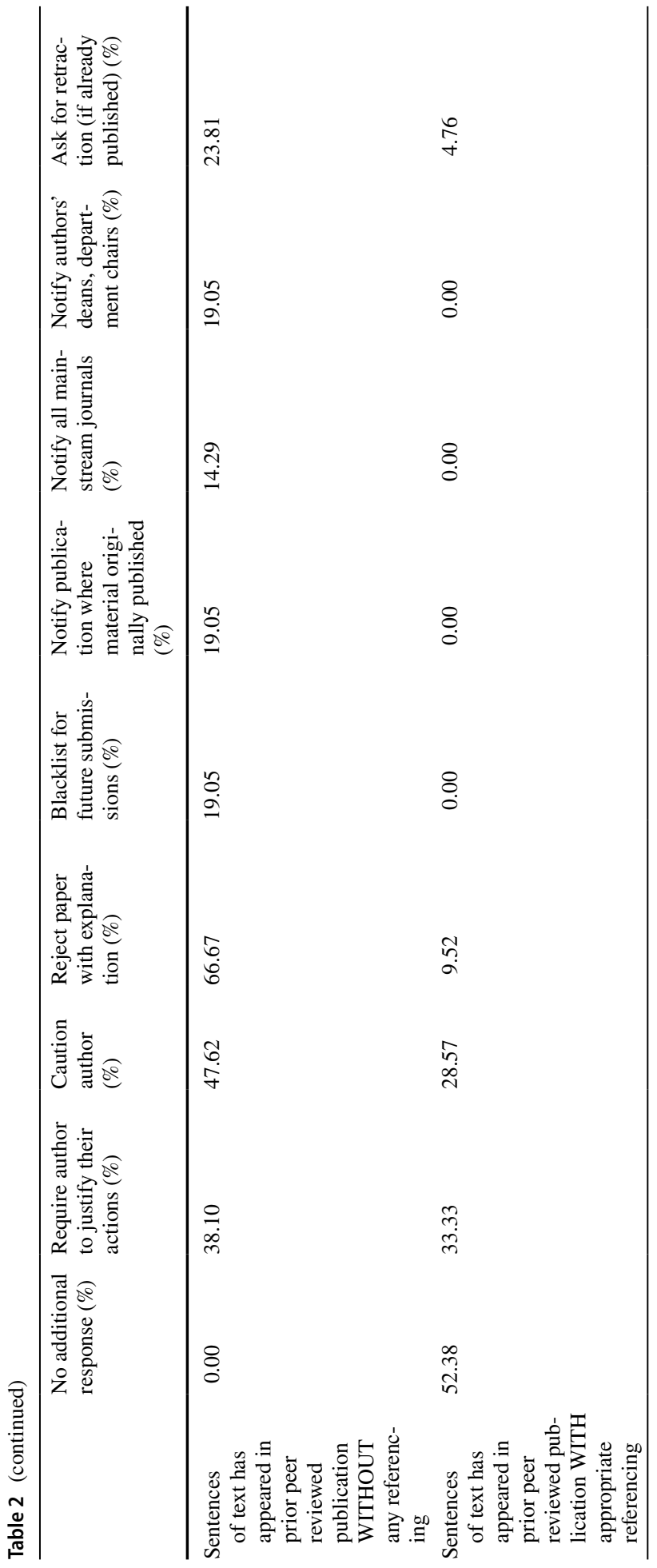




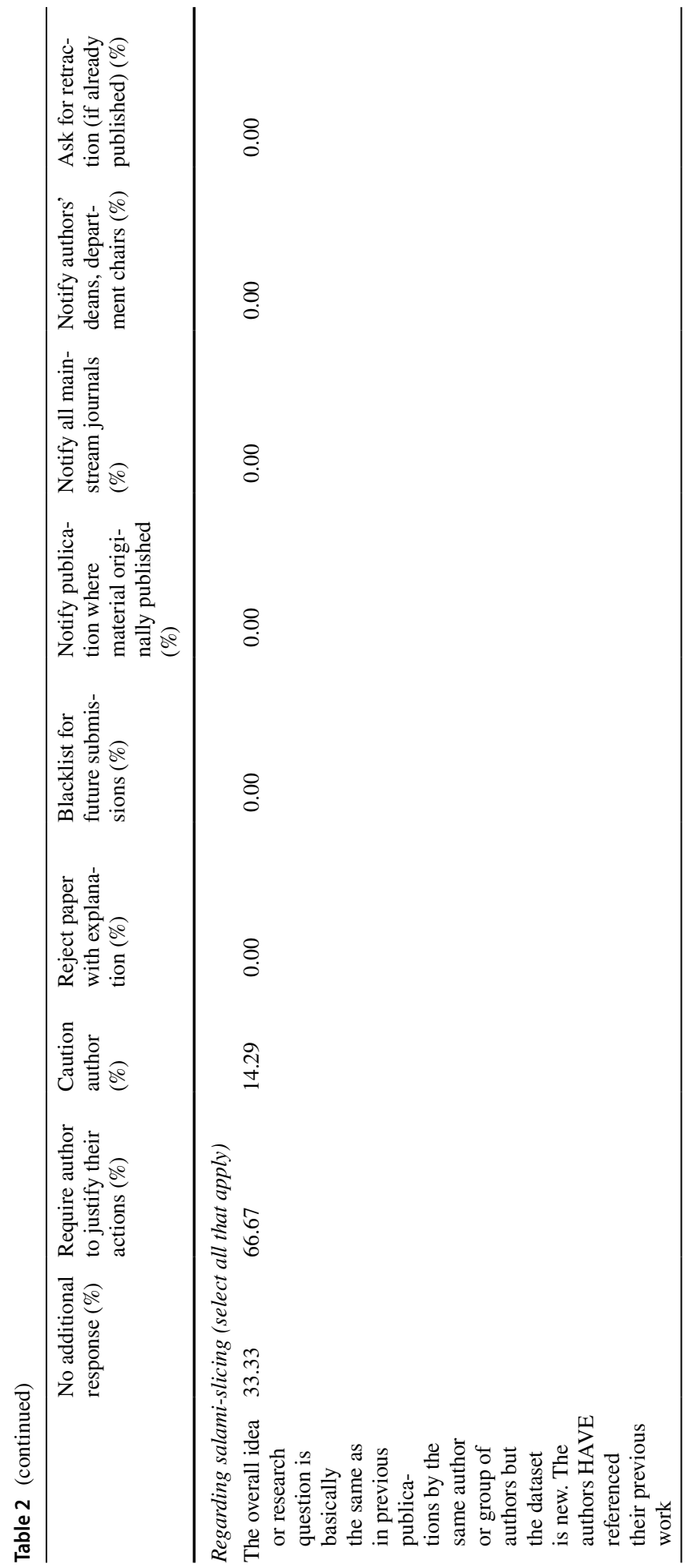




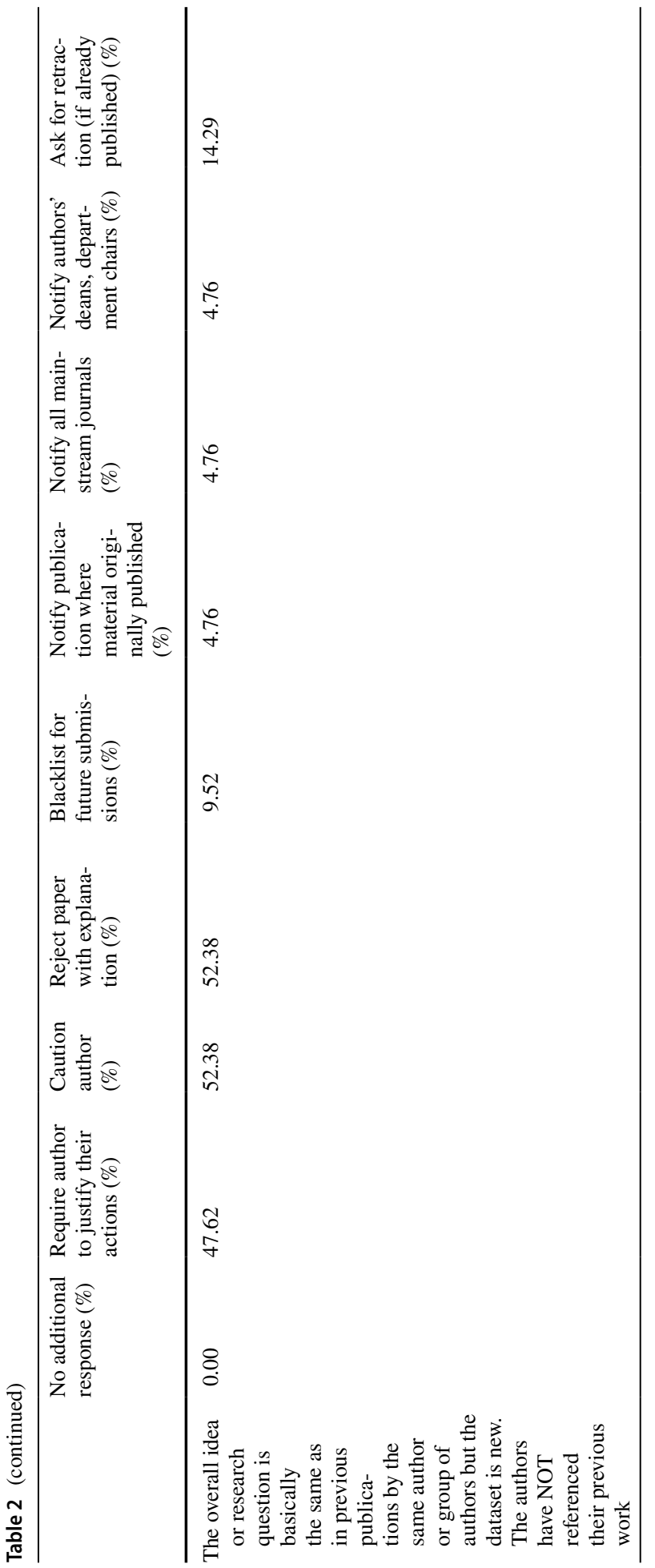




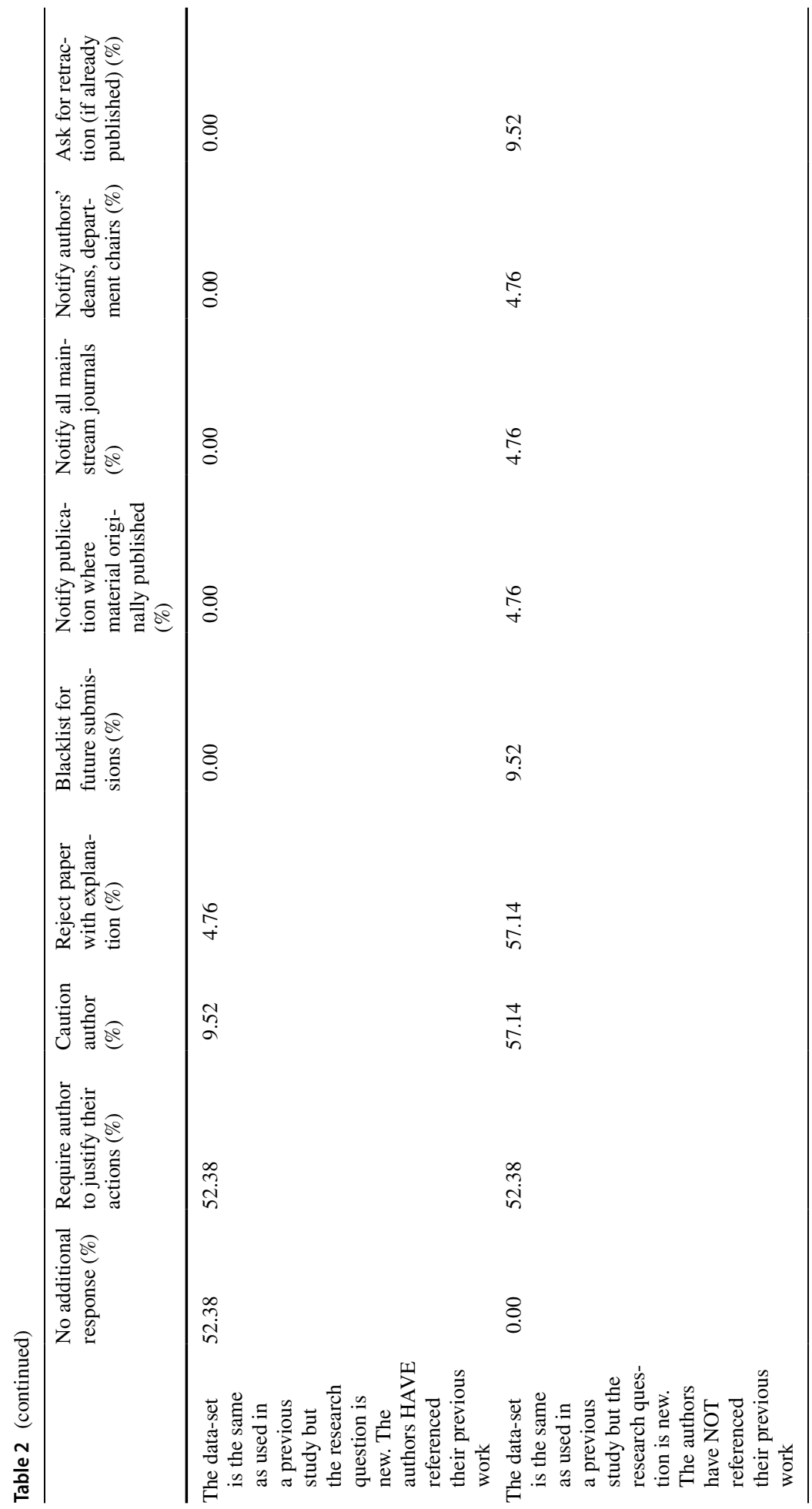


less agreement regarding the severity of consequences that should follow different types of scientific misconduct as well as what should be considered scientific misconduct to begin with. For instance, some editors considered pre-publishing a paper as equivalent to plagiarism whereas other editors did not see it as a problem at all.

The majority of AHSE editors were of the opinion that it is a shared responsibility of the editorial team to carefully evaluate manuscripts for potential scientific misconduct. They expressed a wish to operate within some rules with latitude for individual assessment based on context. However, current guidelines for the assessment and practical handling of scientific misconduct (COPE) may not provide the guidance needed by editors in our field. Instead, some principles may be deducted from our survey results, which may help guide editors as well as authors:

- Authors must declare potential problems with plagiarism, auto-plagiarism and salami-slicing in the cover letter to the editors as well as in the manuscript text.

- Serious consequences (such as blacklisting, contacting authors' deans/department chairs, or retracting published manuscripts) will be considered when authors plagiarize and fail to be transparent about it.

- Auto-plagiarism and salami-slicing on the other hand may result in rejection and cautioning of the authors but should not automatically result in serious consequences such as those mentioned above.

- Reuse of authors' own conference abstracts or text from previously published theses is considered acceptable practice.

- The consequence of plagiarism, auto-plagiarism and salami-slicing should be evaluated individually based on the amount of text or material involved, the extent to which this is acknowledged, and on the type of misconduct committed (with plagiarism being considered more serious than auto-plagiarism and salami-slicing).

- Collectively, editorial teams are responsible for detection of these issues and for formulating an appropriate response.

Given the somewhat fluid and evolving nature of academic publishing, these principles are intended to guide practice and to some extent normalize it, at least within this journal, but they are not expected to replace editorial judgement. We advance them as a guide to those wishing to publish in this journal and as the basis for ongoing debate on what constitutes scientific misconduct in health professions education.

\section{References}

Brice, J., Bligh, J., Bordage, G., Colliver, J., Cook, D., Eva, K. W., et al. (2009). Publishing ethics in medical education journals. Academic Medicine, 84(10 Suppl), S132-4.

COPE Guidelines. Retrieved January 15, 2019 from https://publicationethics.org/resources/flowcharts.

Eva, K. W. (2017). How would you like your salami? A guide to slicing. Medical Education, 51(5), $456-457$.

Gehlbach, H., Artino, A. R., Jr., \& Durning, S. (2010). AM last page: Survey development guidance for medical education researchers. Academic Medicine, 85(5), 925. https://doi.org/10.1097/ ACM.0b013e3181dd3e88.

Norman, G. (2014). Data dredging, salami-slicing, and other successful strategies to ensure rejection: Twelve tips on how to not get your paper published. Advances in Health Sciences Education Practice, 19(1), 1-5. 
Steen, R. G. (2011). Retractions in the scientific literature: Is the incidence of research fraud increasing? Journal of Medical Ethics, 37(4), 249-253.

Publisher's Note Springer Nature remains neutral with regard to jurisdictional claims in published maps and institutional affiliations. 